\title{
An Atlas-Based Segmentation Propagation Framework Using Locally Affine Registration - Application to Automatic Whole Heart Segmentation
}

\author{
Xiahai Zhuang ${ }^{1, *}$, Kawal Rhode ${ }^{2}$, Simon Arridge ${ }^{1}$, Reza Razavi ${ }^{2}$, Derek Hill ${ }^{1}$, \\ David Hawkes ${ }^{1}$, and Sebastien Ourselin ${ }^{1}$ \\ ${ }^{1}$ Centre for Medical Image Computing, Med Phys Dept, UCL, WC1E 6BT, UK \\ ${ }^{2}$ Interdisciplinary Medical Imaging Group, St Thomas' Hospital, KCL, SE1 7EH, UK \\ $\mathrm{x} . \mathrm{zhuang@ucl.ac.uk}$
}

\begin{abstract}
In this paper, we present a novel registration algorithm for locally affine registrations. This method preserves the anatomical and intensity class relationships between the local regions. A regularisation procedure is used to maintain a global diffeomorphic transformation. Combined with a novel generic method for accurately inverting the final deformation field, we include our techniques within an atlas-based segmentation propagation framework. We applied our method to automatically segment the whole heart from cardiac magnetic resonance images from a cohort of 18 volunteers (acquisition resolution 2 $\times 2 \times 2 \mathrm{~mm}$ ). The results show that the proposed method provides a robust initialisation for the atlas-based segmentation propagation framework refined with a fluid registration. We validated our approach against other registration strategies, and demonstrated that we improved the accuracy of the whole heart segmentations $(1.8 \pm 0.42 \mathrm{~mm})$.
\end{abstract}

Keywords: Locally Affine Registration, Inverting Transformations, Whole Heart Segmentation.

\section{Introduction}

Locally affine transformation is an attractive registration alternative for some applications where a single global affine transformation cannot provide enough accuracy while a non-rigid registration would affect incorrectly the local topology. Little et al. proposed a method to incorporate rigid structures into a registration framework. This method registers each of these structures separately and interpolates the local rigid transformations based on a modified distance weighting interpolation [1], [2]. Based on a similar idea, Pitiot et al. proposed a piece-wise affine registration algorithm where sub-images can be automatically extracted prior to individual local registrations [4]. The challenge of these methods is that the priors for extracting the sub-images or structures from both of the target and source images are not always available. Also, the fusion of

* This research has been funded by EPSRC grant GR/T11395/01. 
the local rigid or affine transformations based on the final results does not guarantee a diffeomorphic global deformation field [5].

Commonwick et al. proposed a poly-affine/locally affine registration framework based on a block-matching algorithm using correlation coefficient similarity measure [6]. An interpolation scheme based on fusing the speed of the local transformations is employed to generate a global transformation (poly-affine) from this local set. This algorithm also implicitly provides the inverse transformation and guarantees a diffeomorphism [5]. However, this method computes the similarity for the defined regions separately and optimises the local affine transformations independently. In addition, the fusion of the poly-affine, which involves the complex logarithm of the local matrices, is only used at the final resultant transformations. The more efficient "direct fusion" using distance weighting interpolation is employed during the optimisation iterations. Both the region based optimisation and "direct fusion" make this method still a piece-wise registration technique which loses the intensity class relationship between the regions during the registration procedure.

In this paper, we propose a new Locally Affine Registration Method (LARM). This method preserves the linkage of intensity classes between local regions by computing and optimising a global cost function. A regularisation procedure is used to maintain the diffeomorphism of the global transformation. The registration framework requires a pre-definition of the local regions into the target image. However, in many applications such as atlas-based segmentation propagation, one may only have the definition on the source image. In such cases, a method for computing an inverse transformation from a deformation field is required. Currently reported generic methods have difficulties in dealing with large deformation fields and in controlling maximal errors when the field contains large displacements causing dilations and anisotropic contractions [8]. In this paper, we propose a novel algorithm for inverting transformations based on a modified version of the scatter data interpolation [8]. This method is generic, i.e. widely applicable to any diffeomorphic transformations.

In section 2, we describe in details LARM and the inverse transformation estimation. A qualitative and quantitative analysis of our approach based on phantom data is presented in section 3. In section 4, we apply our algorithms within a segmentation propagation approach for automatic whole heart segmentation from magnetic resonance (MR) images. Conclusion and discussion are given in section 5.

\section{Methodology}

To achieve a global non-linear transformation $T$ from a set of locally affine transformations $\left\{G_{i}\right\}$, we embed our local transformations into a global optimisation scheme. In section 2.1, we propose a novel approach where each $G_{i}$ is computed competitively. We achieve this by optimising a cost function based on the gradient of the normalised mutual information between the local region set $\left\{V_{i}\right\}$. In section 2.2 , we describe our new mathematical framework for inverting a displacement vector field, in order to apply it to a segmentation propagation approach. 


\subsection{Locally Affine Registration Method (LARM)}

Let $\left\{V_{i}\right\}$ be the set of the $n$ pre-defined local regions, $\left\{G_{i}\right\}$ be the set of the $n$ assigned local affine transformations, the direct fusion based on the distance weighting interpolation [1] is:

$$
T(X)=\left\{\begin{array}{ll}
G_{i}(X), & X \in V_{i}, i=1 \ldots n \\
\sum_{i=1}^{n} w_{i}(X) G_{i}\left(X_{i}\right), & X \notin \bigcup_{i=1}^{n} V_{i}
\end{array},\right.
$$

where, $w_{i}(X)$ is the normalised weighting factor related to the distance $d_{i}(X)$ between point $X$ and region $V_{i}$ :

$$
w_{i}(X)=\left(1 /\left(d_{i}(X)\right)^{e}\right) /\left(\sum_{i=1}^{n} 1 /\left(d_{i}(X)\right)^{e}\right) .
$$

In our transformation model, a constant identity affine transformation is assigned to the boundary of the region of interest (ROI) and $e$ is assigned to 2 .

However, two reasons can cause non-diffeomorphism by this direct fusion. Firstly, the local regions can overlap each other after the individual transformations. A correction of the local regions is required to guarantee the non-overlapping:

$$
V_{i}=V_{i}-\left(\bigcup_{i \neq k}\left(\oplus_{l_{i}} R_{i k}\right)\right) \text {, }
$$

where, $R_{i k}=G_{i}^{-1}\left(G_{i}\left(V_{i}\right) \cap G_{k}\left(V_{k}\right)\right)$ are the volumes in $V_{i}$ that will overlap to region $V_{k}$ after the transformations; $\oplus$ is the morphology dilation with length $l_{i}$ to leave enough space for the interpolation to give an anatomically reasonable minimal distance between the regions after the transformation.

Secondly, when the displacement of the local transformation is large the direct fusion by eq. (1) can produce folding [5]. A regularisation step to monitor the Jacobian of the deformation fields in these areas within the volume $V$ of the registration images but outside of all local regions $\left\{V_{i}\right\}$ is then required. The Jacobian $J_{T}$ can be expressed as:

$$
J_{T}=\sum_{i=1}^{n} \frac{\partial w_{i}}{\partial X} \cdot G_{i}+\sum_{i=1}^{n} w_{i} \cdot \frac{\partial G_{i}}{\partial X}=\nabla \mathrm{W} \cdot \mathrm{G}^{\mathrm{T}}+\nabla \mathrm{G} \cdot \mathrm{W}^{\mathrm{T}} .
$$

If the determinant of the Jacobian, $\operatorname{det}\left(J_{T}\right)$, drops below a threshold (e.g. $\operatorname{det}\left(J_{T}\right)<0.5$ as demonstrated in [3]), a new source image is generated by applying the current fusion of local transformations. Hence, the total transformation field are concatenated:

$$
T=T_{m} \circ T_{m-1} \circ \ldots \circ T_{1} .
$$

Since all the $T_{j}$ are diffeomorphic, the composition $T$ keeps this property as well.

The main advantage of the proposed LARM is that the computations of the cost function and the driving force (the derivative of the cost function) are based on the whole image intensity information. To compute the derivative of the normalised mutual information, one can obtain it from the operation of the entropies' derivatives which can be computed from the derivative of the probability density function $p$. Let $(l, k)$ be a joint histogram bin from target image $I_{t}$ and source image $I_{s}, \omega$ be the kernel 
density estimate function, the derivative of $p(l, k)=\sum_{X} \omega\left(I_{t}(X)\right) \cdot \omega\left(I_{s}(T(X))\right)$ against a transformation parameter $\theta_{i}$ of $G_{i}$ is:

$$
\begin{aligned}
\partial p(l, k) / \partial \theta_{i} & =\sum_{X} \omega\left(I_{t}(X)\right) \cdot\left(\partial \omega\left(I_{s}(T(X))\right) / \partial \theta_{i}\right) \\
& =\sum_{X} \omega\left(I_{t}\right) \nabla \omega\left(\nabla I_{s} \cdot \partial G_{i}(X) / \partial \theta_{i}\right) w_{i}(X) \\
& =\sum_{X} W(X)
\end{aligned} .
$$

Given a point $X$ which has a small value of $d_{k}(X)$ that $d_{k}(X) / d_{i}(X)<<1$, then $w_{i}(X)$ tends to be zero, which makes $W(X)$ tend to be zero. For any region $\Omega$, we can rewrite $p(l, k)=A+B$, where $A=\sum_{X \in \bar{\Omega}} \omega\left(I_{t}\right) \omega\left(I_{s}\right)$ and $B=\sum_{X \in \Omega} \omega\left(I_{t}\right) \omega\left(I_{s}\right)$. Therefore, we can also rewrite the derivative of the entropy as:

$$
\frac{\partial H}{\partial \theta_{i}}=-\sum_{l, k} \frac{\partial((A+B) \log (A+B))}{\partial \theta_{i}}=-\sum_{l, k}(1+\log (A+B)) \cdot\left(\frac{\partial A}{\partial \theta_{i}}+\frac{\partial B}{\partial \theta_{i}}\right) .
$$

Let $U_{i}=\left(\oplus_{\lambda}\left(V_{i}\right)\right) \cap V$, where $\lambda$ is the dilation length and large enough to make $w_{i}(X) \approx 0$ when $X \notin U_{i}, V$ is the volume of the images. Let $U_{i}=\Omega$, then $\partial A / \partial \theta_{i} \approx 0$ and:

$$
\frac{\partial H}{\partial \theta_{i}} \approx-\sum_{l, k} \frac{\partial B}{\partial \theta_{i}}(1+\log (A+B)) .
$$

Since $(1+\log (A+B))$ is constant for all $\theta_{i}$, the computation complexity of $(8)$ is $O\left(n \cdot\left|U_{i} \cap V\right|\right)$, which compares with $O(n \cdot|V|)$ of (7). We achieved more than 10 times faster in the segmentation experiments in section 4 by using a value of $40 \mathrm{~mm}$ for $\lambda$.

Fig.1 outlines the framework of the proposed LARM. A global affine is composed with the concatenated $T$ for the global transformation. The regularization step includes the overlap correction and monitoring Jacobian for $T$ concatenation.

For each step of Optimisation

Global transformation, $F(X)=T \circ G$, and $T=T_{m} \circ T_{m-1} \circ \ldots \circ T_{1}$

Optimise global affine $G$

Optimise local affine $G_{i}$ of $T_{m}$, compute the derivative based on eq. (8)

Regularisation step:

For each region $V_{i}$ in $\left\{V_{i}\right\}$

Overlap correction: any point within $l_{i}$ mm from the region $V_{i}$ will be excluded

If exclude points then re-compute the distance transformation of $V_{i}$

End of each region $V_{i}$ in $\left\{V_{i}\right\}$

If $\operatorname{MIN}\left(\operatorname{det}\left(J_{T m}\right)\right)<0.5$ then: new a $T_{m+1}, T=T_{m+1} \circ$, and $m=m+1$

End of Optimisation

Fig. 1. Our proposed Locally Affine Registration Method (LARM) framework described as pseudo-code

\subsection{Inverse Transformation Interpolation}

To compute the inverse transformation $T^{-1}$ from a diffeomorphism, we can directly interpolate $t=T^{-1}(s)$ for every point $s$ in the source image coordinate. To do so, we 
apply the distance weighting interpolation using the closest scatter point set $\left\{s_{i}\right\}$, where $\left\{s_{i}\right\}$ are transformed by $T$ from the point set $\left\{t_{i}\right\}$ in the target image [8].

To compute the approximation $T_{I}(s)$ of the inverse $T^{-1}(s)$, we can interpolate $T_{I}(s)$ using the closest scatter points $\left\{s_{i}\right\}[8]$ :

$$
T_{I}(s)=s+\sum w\left(s_{i}\right) \cdot\left(t_{i}-s_{i}\right),
$$

where, $s_{i}=T\left(t_{i}\right)$, with $t_{i}$ the point from the target image space, and $w\left(s_{i}\right)$ the normalised inverse distances from $s_{i}$ to $s$.

There are two major error sources from this method. Firstly, if the transformation $T$ contains dilations, then the scatter points $\left\{s_{i}\right\}$ can be too distant from $s$. Secondly, if $T$ contains anisotropic contractions, then the points $\left\{t_{i}\right\}$ are too far away from the ground truth $t$. To deal with these issues, instead of only using the closest points forward-transformed from the image grid points of the target image, we propose to use the $2^{d}$ ( $d$-dimensions) closest neighbour points within a voxel size distance to $s$ as the $\left\{s_{i}\right\}$. These points are guaranteed to map from the vertices of a region $O_{i}$, no more than a voxel size, in the target image space. Since both the interpolated point $T_{I}(s)$ and the ground truth $T^{-1}(s)$ are within the region $O_{i}$, the interpolation error $\left|T_{I}(s)-T^{-1}(s)\right|$ should not be larger than the size of $O_{i}$ (hence the voxel size).

Unfortunately, when the transformation $T$ causes local dilations or anisotropic contractions, it can be challenging to find $\left\{s_{i}\right\}$ for $s$. We propose a new method to maximise the probability of finding these points $\left\{s_{i}\right\}$.

When transformation $T$ causes dilation, the vertex points of a voxel $v_{t}$ in the target image will be sparsely transformed into a bigger volume $V_{s}$ in the source image. To obtain accurate interpolation using eq. (9), we propose to resample more points from $v_{t}$. We estimate the number of resample points $S_{\hat{\mathbf{e}}}$ as:

$$
S_{\hat{\mathrm{e}}}=\max (1, \mathrm{I} J: \hat{\mathrm{e}} \mathrm{l}),
$$

where, $J$ is the Jacobian matrix and $|J: \hat{\mathbf{e}}|=|\hat{\mathbf{e}} \times J \times \hat{\mathbf{e}}| . \mid J$ : $\hat{\mathbf{e}} \mid$ represents the volume change in the direction of $\hat{\mathbf{e}}$, where $\hat{\mathbf{e}}$ is the standard $\mathbf{R}^{d}$ Euclidean basis.

When transformation $T$ causes local anisotropic contractions, then $\left|J: \hat{\mathbf{e}}_{j}\right| /\left|J: \hat{\mathbf{e}}_{k}\right|>1$. The vertex points $t_{i}$ mapping to $s_{i}$, closest points of $s$, can be the vertex points of the neighboured voxels of the ground truth $t$ in the direction of $\hat{\mathbf{e}}_{k}$. This case will generate big errors in the inverting transformations. To avoid this, we propose to resample more points in the other orthogonal directions inside $v_{t}$ to provide more accurate point set $\left\{s_{i}\right\}$ for $s$ :

$$
S_{\hat{\mathrm{e}}_{j}}=\prod_{k} \max \left(1,\left|\mathrm{~J}: \hat{\mathrm{e}}_{j}\right| /\left|\mathrm{J}: \hat{\mathrm{e}}_{k}\right|\right), \quad j, k=1,2, \ldots, d .
$$

To combine (10) and (11), one can get the number of resample points inside $v_{t}$ :

$$
S_{\hat{\mathrm{e}}_{j}}=\max \left(1,\left|J: \hat{\mathrm{e}}_{j}\right|\right) \cdot \prod_{k} \max \left(1,\left|J: \hat{\mathrm{e}}_{j}\right| /\left|J: \hat{\mathrm{e}}_{k}\right|\right), j, k=1,2, \ldots, d .
$$

\section{Phantom Study}

Fig. 2 shows a phantom data registration task which demonstrates the advantage of the proposed LARM over the region-based registration method (RBRM) [9]. RBRM 
extracts the regions (two bars) from the target image into independent sub-images to separately align them and to derive the local affine transformations. We can see from Fig. 2 (d) and (e) that LARM succeeds, while RBRM fails. This is due to the large distance between the two corresponding right bars and the lack of intensity information linkage between the sub-images of the target image in local registrations. Further quantitative comparisons using real data can be found in section 4 .

To study the performance of the proposed inverting method, we generate a source and a target image with an isotropic voxel size of $0.5 \mathrm{~mm}$. We create a local rigid box region at the centre of the target image which can be rotated. We fixed the boundaries of the target image. By rotating the box from 5 to 85 degrees, using eq. (1) for interpolation, we can generate a set of generic transformation fields. Each transformation from this set includes a rigid rotation in the centre and some nonlinear dilations and contractions in the other area. Fig. 3 (a) presents the inverse-consistency (IC) errors (I $\left.T\left(T_{I}(s)\right)-s \mid\right)$ of inverting these transformation fields. We can see that the mean IC errors and the maximal IC errors are subvoxel, which is not the case for most of the currently reported methods [8]. In addition, the maximal IC error reaches its largest value at a 45-50 degrees rotation. This indicates that the IC error is less affected by the magnitude of displacements in large transformation fields.

A common source of errors can arise from scaling up and down objects. To study the behaviours of our algorithm, we scale up the target image along one direction ( $\mathrm{X}$ axis) from 1.5 to 4.5 times (the magnitudes of transformations are from $6 \mathrm{~mm}$ to $36 \mathrm{~mm}$ ), and scale down (along $\mathrm{X}$ axis) the image from $1 / 0.9$ to $1 / 0.2$ times ( $3 \mathrm{~mm}$ to $8 \mathrm{~mm}$ ). The maximal IC errors by scaling up transformations are more stable than by scaling down transformations as shown in Fig. 3 (b), even though the transformation magnitudes of the scaling down are much smaller. All the maximal IC errors in the two studies are subvoxel (less than $0.5 \mathrm{~mm}$ ).

\section{Whole Heart Segmentation}

We include our techniques within an atlas-based segmentation propagation framework. When the atlas and MR image are from different subjects, the locally affine transformation model provides a better initialisation than a global affine transformation. In this section, we employ nineteen volunteer data sets, acquired in St Thomas' Hospital, London, to demonstrate the advantage of a good initialisation in the segmentation framework as well as the robustness and accuracy of LARM. The accuracy is computed from the surface distances between segmentation results and their ground truth. The MR images are from the balanced steady state free precession for whole heart imaging with acquisition resolution $2 \times 2 \times 2 \mathrm{~mm}$ at end-diastolic phase. The images are reconstructed into $1 \times 1 \times 1 \mathrm{~mm}$ voxel dimensions to achieve better surface delineations in their segmentations. The segmentation ground truth labels every anatomical region with different intensity value to produce an atlas. They have been done manually by technical experts and double-checked by cardiologists.

We select one MR image and its atlas, containing seven labelled regions, as shown in Fig.4. We then apply a segmentation propagation framework to the other $18 \mathrm{MR}$ images, using the fluid registration algorithm as a final refinement [7]. The seven 


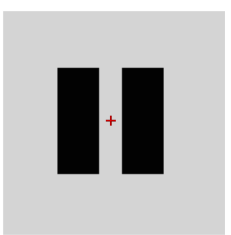

(a)

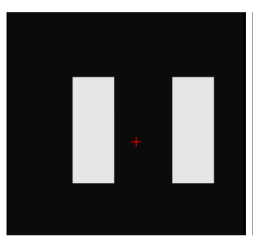

(b)

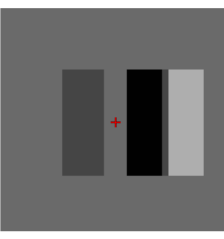

(c)

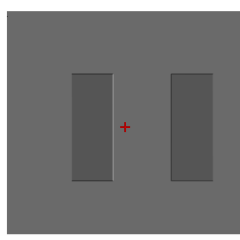

(d)

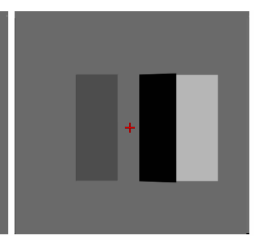

(e)

Fig. 2. The source image (a) and the target image (b) used in the registration phantom study; (c) is the mixed intensity image of the target image and the source image. (d) is the mixed image of the target image and the resultant image by LARM which succeeds; (e) is the mixed image of the target image and the resultant image by RBRM which fails.

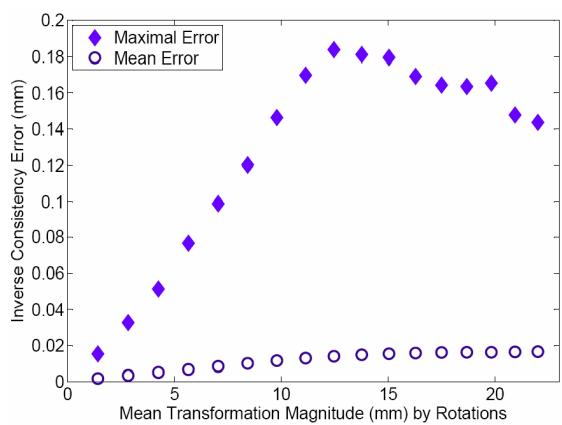

(a)

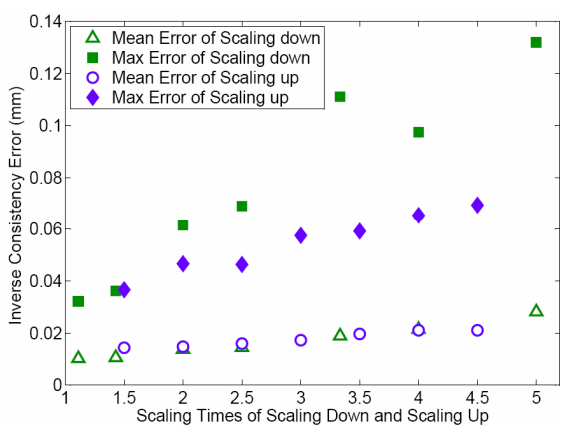

(b)

Fig. 3. The scaling of the inverse-consistency error with applied rotation (a) and scaling (b)
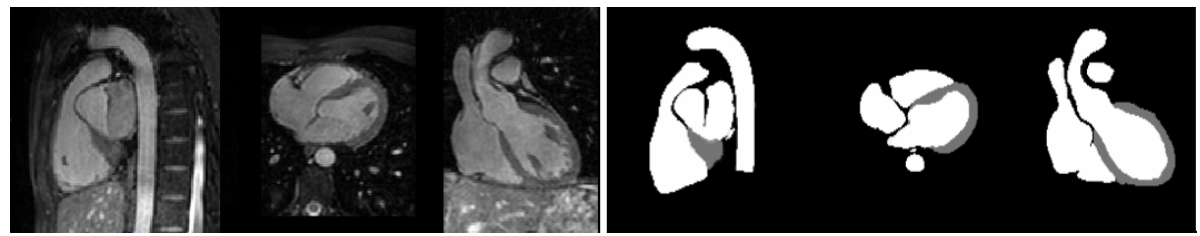

Fig. 4. The three views of the cardiac MR image and its manual segmentation labels

Table 1. The segmentation errors by the five methods on the five surfaces and the whole heart segmentation (SGAR: single global affine registration; RBRM: region-based registration method; LARM: locally affine registration method; FGAR: fluid with global affine registration; FLAR: fluid with the locally affine registration)

\begin{tabular}{cccccc}
\hline Unit $(\mathrm{mm})$ & SGAR & RBRM & LARM & FGAR & FLAR \\
\hline Left Ventricle & $5.9 \pm 1.3$ & $3.6 \pm 1.3$ & $4.4 \pm 1.4$ & $3.3 \pm 2.0$ & $2.4 \pm 1.1$ \\
Right Ventricle & $4.0 \pm 2.3$ & $3.8 \pm 3.9$ & $3.2 \pm 1.5$ & $3.3 \pm 3.7$ & $2.6 \pm 1.5$ \\
Left Atrium & $4.7 \pm 1.6$ & $3.5 \pm 3.1$ & $2.9 \pm 1.0$ & $3.0 \pm 2.0$ & $1.9 \pm 0.78$ \\
Right Atrium & $5.1 \pm 2.5$ & $3.7 \pm 2.6$ & $3.2 \pm 2.1$ & $4.3 \pm 4.7$ & $2.4 \pm 2.7$ \\
Epicardial & $4.4 \pm 2.1$ & $1.6 \pm 0.85$ & $1.7 \pm 0.32$ & $2.3 \pm 1.7$ & $1.3 \pm 0.21$ \\
Whole Heart & $4.1 \pm 1.6$ & $2.9 \pm 1.6$ & $2.8 \pm 0.40$ & $2.7 \pm 1.7$ & $1.8 \pm 0.42$ \\
\hline
\end{tabular}


regions, including four chambers (myocardium included in left ventricle) and two major vessels (aorta is divided into two) are used as the pre-defined local regions for LARM and RBRM. We assess five methods: the single global affine registration (SGAR), RBRM, LARM, the fluid with global affine registration (FGAR), and the fluid with the locally affine registration (FLAR) on four chambers' endocardial surfaces, left ventricle's epicardial surfaces, and the whole heart segmentation. These surfaces have been manually delineated in all 19 data sets.

Table. 1 presents the results of the segmentation accuracy. We can see that LARM achieves better accuracy and robustness (lower standard deviation) than the other two initialisation methods (SGAR and RBRM). We conclude from this study that the proposed approach improves the segmentation-propagation framework by providing a robust initialisation of the atlas.

\section{Conclusion and Future Work}

In this paper, we have proposed a new framework for locally affine registrations and a novel inverting transformation technique. We have applied these techniques to an atlas-based segmentation framework for automatic whole heart segmentations from cardiac MR images. Both the average and standard deviation of the accuracy have been improved by the locally affine registration method, compared with the single global affine registration and region-based registration.

In future work, we would like to improve the nonrigid registration to preserve the geometric (anatomical) constraints. Indeed, this lack of constraints can result in higher standard deviation when the region boundaries are not initialised closely enough. We would also like to apply the segmentation framework to pathological data, such as from hearts with severely dilated ventricles or congenital malformations, which currently still challenge most of the model based segmentation methods.

\section{References}

1. Shepard, D.: A Two-dimensional Interpolation Function for Irregularly-spaced Data. In: National Conference of the ACM, pp. 517-527. ACM Press, New York (1968)

2. Little, J.A., Hill, D.L.G., Hawkes, D.J.: Deformations Incorporating Rigid Structures. Computer Vision and Image Understanding 66(2), 223-232 (1997)

3. Bro-Nielsen, M., Gramkow, C.: Fast Fluid Registration of Medical Images. In: Höhne, K.H., Kikinis, R. (eds.) VBC 1996. LNCS, vol. 1131, pp. 267-276. Springer, Heidelberg (1996)

4. Pitiot, A., Malandain, G., Bardinet, E., Thompson, P.M.: Piecewise Affine Registration of Biological Images. In: Gee, J.C., Maintz, J.B.A., Vannier, M.W. (eds.) WBIR 2003. LNCS, vol. 2717, pp. 91-101. Springer, Heidelberg (2003)

5. Arsigny, V., Pennec, X., Ayache, N.: Polyrigid and Polyaffine Transformations: A Novel Geometrical Tool to Deal with Non-rigid Deformations-Application to the Registration of Histological Slices. Medical Image Analysis 9(6), 507-523 (2005) 
6. Commowick, O., Arsigny, V., Costa, J., Ayache, N., Malandain, G.: An Efficient Locally Affine Framework for the Registration of Anatomical Structures. In: ISBI, pp. 478-481 (2006)

7. Crum, W.R., Hill, D.L.G., Hawkes, D.J.: Information Theoretic Similarity Measures in Non-rigid Registration. In: Taylor, C.J., Noble, J.A. (eds.) IPMI 2003. LNCS, vol. 2732, pp. 378-387. Springer, Heidelberg (2003)

8. Crum, W.R., Camara, O., Hawkes, D.J.: Methods for Inverting Dense Displacement Fields: Evaluation in Brain Image Registration. In: Ayache, N., Ourselin, S., Maeder, A. (eds.) MICCAI 2007, Part I. LNCS, vol. 4791, pp. 900-907. Springer, Heidelberg (2007)

9. Zhuang, X., Hawkes, D.J., Crum, W., Boubertakh, R., Uribe, S., Atkinson, D., Batchelor, P., Schaeffter, T., Razavi, R., Hill, D.L.G.: Robust registration between cardiac MRI images and atlas for segmentation propagation. In: SPIE-MI, vol. 6914, p. 6914, 07 (2008) 